\title{
Expression of ERO1L in gastric cancer and its association with patient prognosis
}

\author{
BO ZHOU, GONGPING WANG, SHEGAN GAO, YE CHEN, CANHUI JIN, ZENGFANG WANG, \\ YANTONG YANG, ZHIKUN MA, WEI ZHANG and XIAOSHAN FENG
}

\begin{abstract}
Department of Oncology Surgery, The First Affiliated Hospital and College of Clinical Medicine of Henan University of Science and Technology, Luoyang, Henan 471003, P.R. China
\end{abstract}

Received October 12, 2016; Accepted April 11, 2017

DOI: $10.3892 /$ etm.2017.4782

\begin{abstract}
The present study aimed to assess the expression of endoplasmic reticulum oxidoreductin-1-like (ERO1L) in gastric cancer and determine its association with patient prognosis. A total of 105 patients with gastric cancer undergoing radical gastrectomy were selected for the current study. Gastric cancer tissues (the observation group) and normal gastric tissue adjacent to the carcinoma (the control group) were resected from patients. Levels of ERO1L mRNA and protein in tumor tissues and adjacent tissues were detected using reverse transcription-quantitative polymerase chain reaction, western blotting and immunohistochemistry. Patients were divided into two groups: A positive group and negative group, according to the expression of ERO1. The expression of ERO1L in gastric cancer and its association with patient prognosis was analyzed. Levels of ERO1 mRNA and protein in gastric cancer were significantly higher than those of adjacent tissues $(\mathrm{P}<0.05)$. Immunohistochemical analysis demonstrated that there were 22 patients exhibiting negative expression of ERO1L and 83 patients exhibiting positive expression of ERO1L. The cumulative recurrence rates over 3 years in patients with positive expression of ERO1L were significantly higher than in patients with negative expression of ERO1L $(\mathrm{P}<0.05)$; the cumulative survival rates over 3 years in patients with positive expression of ERO1L were significantly lower than those of patients with negative expression of ERO1L $(\mathrm{P}<0.05)$. Thus, the current study determined that ERO1L was highly expressed in gastric cancer tissue. The high expression of ERO1L was associated with adverse prognoses in patients
\end{abstract}

Correspondence to: Professor Xiaoshan Feng, Department of Oncology Surgery, The First Affiliated Hospital and College of Clinical Medicine of Henan University of Science and Technology, 24 Jinhua Road, Luoyang, Henan 471003, P.R. China

E-mail: xiaoshanfenghn@126.com

Key words: gastric cancer, endoplasmic reticulum oxidoreductin-1-like, prognosis, cumulative survival rate, cumulative recurrence rate with gastric cancer. ERO1L may therefore be a therapeutic target for the prevention of gastric cancer.

\section{Introduction}

Gastric cancer is the second most common malignant tumor and has the second highest cancer-associated mortality rate in the world and the highest cancer-associated mortality rate in China (1). The morbidity and mortality rates of gastric cancer are increasing, posing an increasing threat to public health (2). The symptoms of gastric cancer are mostly non-specific and this mainly accounts for its delay in presentation. The prognosis of patients with gastric cancer is closely associated with its diagnosis and treatment. The methods used to diagnose gastric cancer include endoscope, ultrasound and immunological detection (3). At present, there are multiple chemotherapy regimens used for gastric cancer, which will be chosen according to the patient's condition and physical fitness (4). If standardized comprehensive treatment is provided when patients are in the earlier stages of gastric cancer, the 5-year survival rate may be $>90 \%$ and some patients enter remission. By contrast, although standardized comprehensive treatment is performed on patients with advanced gastric cancer, the 5-year survival rate remains $<5 \%(5,6)$. In China, $70 \%$ of patients with gastric cancer are in the middle or advanced stages when a definitive diagnosis is made. These patients are unable to undergo tumor resection and require chemotherapy instead. However, chemotherapy regimens often fail in such patients, as gastric cancer cells readily develop multidrug resistance $(7,8)$. Thus, promoting the early detection, diagnosis and treatment of gastric cancer is key to improving the rates of remissio'n and survival in patients with gastric cancer. Additionally, it is vital to clarify the pathogenesis of gastric cancer to improve the treatment and prognosis of patients.

The endoplasmic reticulum oxidoreductin-1-like protein (ERO1L) gene is located on chromosome 14 in humans and is considered to be the primary source of endoplasmic reticulum (9). ERO1L protein is an oxidase in the endoplasmic reticulum which regulates hypoxia-induced oxidative protein folding. ERO1L may catalyze the formation of a protein folding disulfide bond and is thought to be involved in cellular apoptosis and tumor infiltration, as well as metastasis induced by endoplasmic reticulum stress (ERS) (10-12). The current 
study assessed the expression of ERO1L in gastric cancer and analyzed the association between ERO1L expression and the prognosis of patients with gastric cancer, so as to elucidate the value of ERO1L protein in postoperative prognosis of gastric cancer.

\section{Patients and methods}

Patients. A total of 105 patients with gastric cancer undergoing radical gastrectomy at The First Affiliated Hospital of Henan University of Science and Technology (Henan, China) were recruited in the current study between October 2013 and October 2015. There were 63 males and 42 females, with mean age of $53.7 \pm 12.8$ years old (range, $39-75$ years). Gastric cancer tissue and normal gastric tissue adjacent to the carcinoma were taken from all patients during radical resection of gastric tumors. Lesions were not detected in normal adjacent tissues following pathological examination. The tumor location in each patient varied. There were 46 cases in the gastric antrum/pylorus, 36 cases in gastric fundus/cardia and 23 cases in the gastric body. Additionally, the tumor-node-metastasis (TNM) stages of all patients were assessed (13). There were 46 patients with TNM stage II and 59 patients had stage III. The current study was conducted in accordance with the declaration of Helsinki and was approved by the Ethics Committee of the First Affiliated Hospital of Henan University of Science and Technology (Henan, China). Written informed consent was obtained from all participants.

$R T$-PCR. A total of $0.1 \mathrm{~g}$ tissue was weighed and TRIzol (Takara, Biotechnology, Co., Ltd., Dalian, China) was added. The tissue was cut into small pieces using surgical scissors and ground using a glass homogenizer. Total RNA was extracted using TRIzol reagent (Thermo Fisher Scientific, Inc., Waltham, MA, USA) according to the manufacturer's instructions. The final precipitation was dissolved in RNase free water, the optical density (OD) value of total RNA and $\mathrm{OD}_{260} / \mathrm{OD}_{280}$ were recorded to calculate RNA purity. RNA was transcribed into cDNA using the PrimeScript RT Reagent kit (Takara Biotechnology, Co., Ltd.), following the manufacturer's instructions. GAPDH was used as a reference gene. The primers used were as follows: ERO1L, forward, 5'-CCATTA GTGCTGCCA-ACCAGTA-3' and reverse, 5'-ATCTGCATC AGCATCACGGTC-3'; GAPDH, forward, 5'-AGAAGGCTG GGGCTCATTTG-3' and reverse, 5'-AGGGGCCATCCACAG TCTTC-3'. The primer was diluted to $10 \mu \mathrm{mol} / \mathrm{l}^{-1}$. A PCR reaction system (Bio-Rad Laboratories, Inc., Hercules, CA, USA) was prepared with a total reaction volume of $20 \mu$ l. The PCR reaction conditions were as follows: Initiation for $3 \mathrm{~min}$ at $94^{\circ} \mathrm{C}$, degeneration for $30 \mathrm{sec}$ at $94^{\circ} \mathrm{C}$, annealing for $30 \mathrm{sec}$ at $60^{\circ} \mathrm{C}$ and extension for $30 \mathrm{sec}$ at $72^{\circ} \mathrm{C}$, for a total of 35 cycles. The data were directly read from the ABI 7500 Real-time PCR system (Applied Biosystems; Thermo Fisher Scientific, Inc.) and quantitatively analyzed using the $2^{-\Delta \Delta \mathrm{Cq}}$ method (14).

Western blotting. A total of $0.15 \mathrm{~g}$ gastric cancer and normal tissue was weighed and cut into pieces using the scissors. The sample was ground using the glass homogenizer, washed using $10 \mathrm{X}$ pre-cooled PBS, centrifuged at $3,000 \mathrm{x}$ g for $5 \mathrm{~min}$ at $4^{\circ} \mathrm{C}$. The supernatant was removed, radioimmunoprecipitation assay buffer (Beyotime Institute of Biotechnology, Shanghai, China) was placed in the ice box, lyzed for $30 \mathrm{~min}$ and centrifuged at $15,000 \mathrm{x} \mathrm{g}$ for $10 \mathrm{~min}$ at $4^{\circ} \mathrm{C}$. The supernatant was removed and protein concentration was determined using the BCA protein assay kit (Beyotime Institute of Biotechnology), $5 \mathrm{X}$ SDS sample buffer was added; the final concentration was $1 \mathrm{X}$. The sample was boiled for $30 \mathrm{~min}$ prior to $12 \%$ SDS-PAGE (100 ng protein per lane). The protein was then transferred onto PVEF membranes and blocked with 5\% nonfat milk powder for $30 \mathrm{~min}$ at room temperature. Mouse anti human ERO1L monoclonal antibody (dilution, 1:1,000; cat. no. ab57177; Abcam, Cambridge, UK) was added, incubated overnight at $4^{\circ} \mathrm{C}$, prior to washing with PBST three times for 5 min each time. Goat-anti-mouse immunoglobulin G monoclonal antibody (dilution, 1:2,000; cat. no. sc-2005; Santa Cruz Biotechnology, Inc., Dallas, TX, USA) was added and incubated at room temperature for $1 \mathrm{~h}$. The 3,3'-diaminobenzidine (DAB) reagent (Beyotime Institute of Biotechnology) was used for visualization and photographed. GAPDH antibody (dilution, 1:2,000; cat. no, sc-25778; Santa Cruz Biotechnology, Inc.) was used as an internal reference, and incubated overnight at $4^{\circ} \mathrm{C}$. Results were analyzed using the Image $\mathrm{J}$ software (version 1.38, National Institutes of Health, Bethesda, MD, USA) and the relative expression of target protein was calculated.

Immunohistochemical analysis. The tissue specimens, including controls and gastric cancer tissue, were embedded in paraffin using embedding agent at room temperature for $1 \mathrm{~h}$, continuously cut into $4 \mathrm{~mm}$ slices, adhered on a glass slide processed with polylysine, baked in an oven at $50^{\circ} \mathrm{C}$ for $1 \mathrm{~h}$, hydrated using xylene and 100, 95, 80 and 75\% ethanol and washed three times with distilled water. The slides were then arranged in the buffer solution containing sodium citrate, heated twice for 8 min each time and washed with PBS three times. Slides were then incubated in $3 \% \mathrm{H}_{2} \mathrm{O}_{2}$ solution at room temperature for $10 \mathrm{~min}$ to block endogenous catalase activity and followed by three washes with PBS 5 min each time. The ERO1L antibody (dilution, 1:100; cat. no. ab57177) was added, incubated overnight at $4^{\circ} \mathrm{C}$ and washed 3 times using PBST for 5 min each time. Subsequently, 10\% goat-anti-mouse secondary antibody (dilution, 1:2,000; cat. no. sc-2005; Santa Cruz Biotechnology, Inc.) was added, incubated at room temperature for $1 \mathrm{~h}$ and washed for 3 times using PBST for 5 min each time. DAB development, staining with hematoxylin, $0.1 \%$ hydrochloric acid differentiation, dehydration, transparency and neutral resin sealing were performed successively. A fluorescent microscope (BX61 Automated Fluorescent Microscope; Olympus Corporation, Tokyo, Japan) was used to observe the above results at magnification, $\mathrm{x} 200$.

Observation index. Microscope observation demonstrated that ERO1L protein was primarily detected in the cytoplasm. The staining was pale yellow and brown and cells were analyzed according to cell staining intensity. For each tissue section, 5 high power fields were selected. A total of 100 target cells were counted under each high power field. The average percentage of 5 fields was judged as the result. Staining evaluation was performed as follows: 0 , all negative; 1 , positive cells $\leq 10 \%$; 2 , positive cells $11-50 \% ; 3$, positive cells $51-75 \%$; 4 , positive 


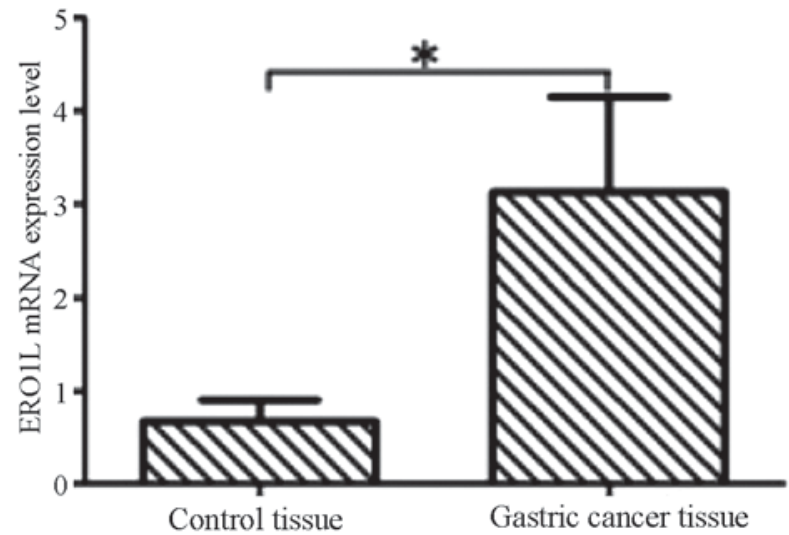

Figure 1. Analysis of ERO1L mRNA levels in control and gastric cancer tissues by reverse transcription-quantitative polymerase chain reaction. Data are presented as the mean + standard deviation. ${ }^{*} \mathrm{P}<0.05$. ERO1L, endoplasmic reticulum oxidoreductin-1-like.

cells $>75 \%$. Staining intensity was evaluated as follows: 0 , no staining; 1 , pale yellow; 2 , brown; 3 , deep yellow. The two scores were multiplied and the total score was the demarcation point. $<4$ was determined to indicate negative ERO1L expression and $\geq 4$ was determined to indicate positive expression of ERO1L. The cumulative survival and recurrence rates were evaluated in the ERO1L positive and negative groups.

Statistical analysis. All data were analyzed using SPSS 17.0 statistical software (SPSS, Inc, Chicago, IL, USA). All measurement data are presented as the mean \pm standard deviation. The measurement data among groups were compared using the Student's t-test. The cumulative survival and recurrence rates were analyzed using the log-Rank test. $\mathrm{P}<0.05$ indicated a statistically significant difference.

\section{Results}

Comparison of EROIL mRNA levels between the two groups of tissue. The results of RT-qPCR indicated that ERO1L mRNA levels in gastric cancer tissues were significantly higher compared with normal adjacent tissues and that this difference was statistically significant $(\mathrm{P}<0.05$; Fig. 1).

Comparison of EROIL protein expression in the two groups of tissue. The results of the western blot analysis demonstrated that, compared with adjacent tissues, ERO1L expression in gastric cancer tissue was significantly higher ( $\mathrm{P}<0.05$; Fig. 2).

EROIL protein expression in the two groups of tissues. As presented in Fig. 3, the results of immunohistochemistry demonstrated that ERO1L was primarily expressed in the cytoplasm, but was not expressed in the nucleus or cell membrane. Results from immunohistochemistry demonstrated that expression of ERO1L was negative in the adjacent tissues, whereas its expression was markedly increased in gastric cancer tissues. In gastric cancer tissue, the expression of ERO1L was positive in 83 cases and negative in 22 cases.

Expression of EROIL and its association with the prognosis of patients. As presented in Fig. 4, all patients were followed up for $>36$ months. Kaplan-Meier curve indicated that the cumulative recurrence rates at 1,2 and 3 years were $25.30 \%(n=21)$, $38.55 \%(n=32)$ and $48.19 \%(n=40)$ in ERO1L positive patients; The cumulative recurrence rates within 1, 2 and 3 years were $13.64 \%(n=3), 22.73 \%(n=5)$ and $31.82 \%(n=7)$ respectively in ERO1L negative patients. The cumulative recurrence rate of ERO1L positive patients was significantly higher than that of negative patients, and the difference was statistically significant $(\mathrm{P}<0.05$; Fig. 4A). Log-Rank test indicated that the cumulative survival rates for 1,2 and 3 years were $75.90 \%$ $(n=63), 63.86 \%(n=53)$ and $49.40 \%(n=41)$ respectively in patients positive for ERO1L. By contrast, the cumulative survival rates for 1, 2 and 3 years were $90.91 \%(n=20), 81.82 \%$ $(n=18)$ and $72.73 \%(n=16)$ respectively, in patients negative for ERO1L. The cumulative survival rate in patients positive for ERO1L was significantly lower than that of patients negative for ERO1L ( $\mathrm{P}<0.05$; Fig. 4B).

\section{Discussion}

Gastric cancer is a type of malignant tumor originating from the mucosal epithelial cells of the gastric wall surface layer and can occur in various different areas of the stomach (15). In China, the incidence and mortality rates of gastric cancer are the highest of all malignant tumors. The clinical manifestations of early gastric cancer are mucosal dysplasia and intestinal metaplasia, associated with chronic atrophic gastritis, and have poor specificity, which do not attract the attention of patients and clinicians (16). The majority of patients are in the middle and advanced stages of gastric cancer when they are diagnosed, which is often accompanied by infiltration of adjacent tissues and organs or varying degrees of distant metastasis. In China, most patients generally visit a doctor when marked symptoms occur, which usually occurs when patients are in the middle and advanced stage of gastric cancer. Therefore, the diagnosis rate of patients with early gastric cancer accounts for $<10 \%$ of all gastric cancer diagnoses in China (17-19). At present, treatments for gastric cancer primarily rely on surgical treatment, combined with radiotherapy and chemotherapy and marked progress has been made in the efficacy of treatments. However, the long-term survival rate of patients remains low and patient quality of life is low. The mechanisms responsible for the occurrence, development and prognosis of gastric cancer are still not fully understood. The present data indicate that they are closely associated with a number of factors. Therefore, it is important to identify a novel molecular target for clinical treatment and curative effect prediction $(20,21)$. Previous studies determined that endoplasmic reticulum oxide (ERO1L) may be used as a potential marker of gastric cancer (22). Therefore, the role of ERO1L in the occurrence and development of gastric cancer was assessed in the current study.

The endoplasmic reticulum is the primary organelle of the cell and its primary function is to be responsible for the folding of protein and to maintain cell homeostasis (23). The stimulation of certain physiological, biochemical and pathological factors can lead to oxidative stress, so as to affect protein folding, the accumulation of unfolded protein and the misfolding of proteins in the endoplasmic reticulum, which leads to ERS $(21,24)$. It was previously determined that ERS was prevalent in tumor tissues (25). ERS was positively 


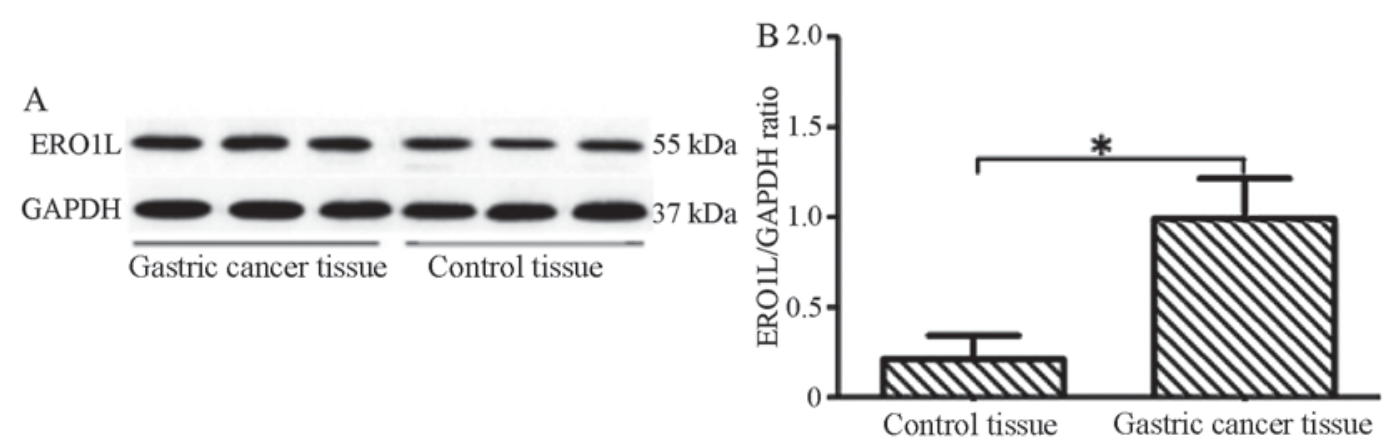

Figure 2. Comparison of ERO1L expression in the two groups of tissue. (A) Western blots indicating ERO1L expression in the gastric cancer and adjacent normal tissue. (B) ERO1L expression in the two groups as determined by quantitative analysis. Data are presented as the mean \pm standard deviation. "P<0.05. ERO1L, endoplasmic reticulum oxidoreductin-1-like.
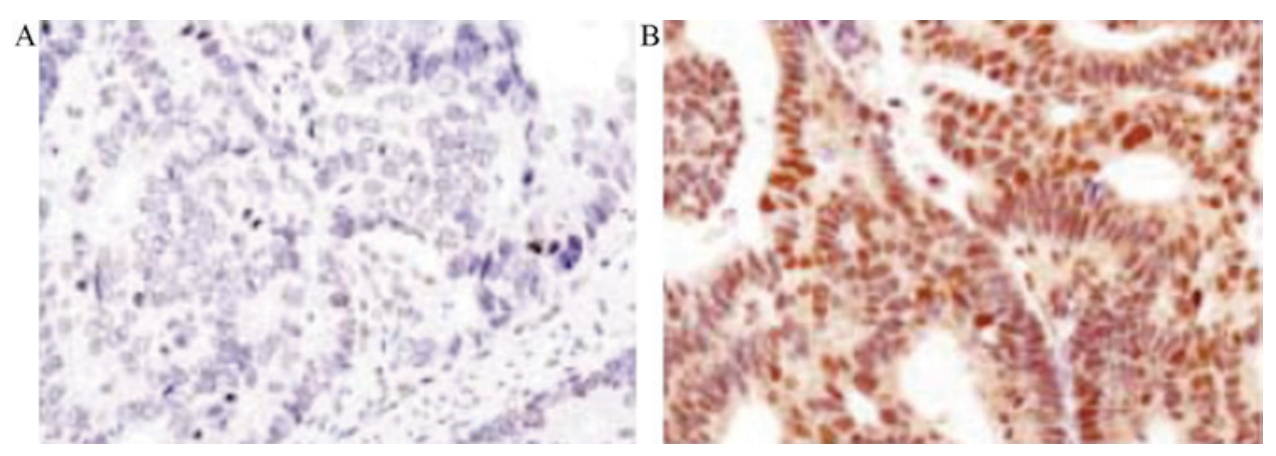

Figure 3. Immunochemical assay results of endoplasmic reticulum oxidoreductin-1-like expression in the two groups of tissue. (A) Control tissue and (B) gastric cancer tissue. Magnification, x200.
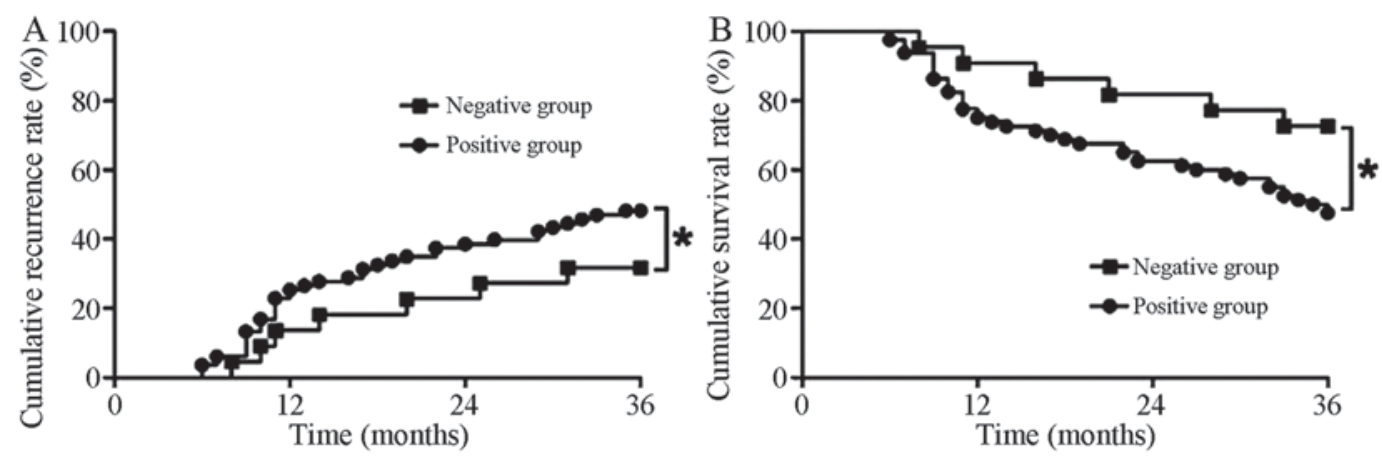

Figure 4. The cumulative recurrence rate and survival rate over 12, 24 and 36 months in patients who were ERO1L negative compared with those who were ERO1L positive. (A) Cumulative recurrence rate curve and (B) Cumulative survival rate curve. ${ }^{*} \mathrm{P}<0.05$. ERO1L, endoplasmic reticulum oxidoreductin-1-like.

correlated with the depth of tumor invasion and degree of metastasis, indicating that ERS might be an important inducement of invasion and metastasis of tumor cells (26). ERS, a type of self-protective reaction of the cells, is involved in the occurrence and development of breast, liver and pancreatic cancer (27-29). ERO1L may promote the formation of a disulfide bond of unfolded protein and can maintain the appropriate oxidation environment of the endoplasmic reticulum as a redox sensor (30). It was determined that the appropriate inhibition of ERO1L may promote the transduction of an anti-severe ERS signal. Thus, ERO1L serves an important role in maintaining the function of ER and comprehensive abilities $(10,31)$. In the present study, the expression of ERO1L in gastric cancer and adjacent normal tissue was determined by RT-qPCR and western blotting. The results determined that the expression of ERO1L in gastric cancer tissue was significantly higher than in adjacent tissues both at the mRNA and protein levels. In addition, immunofluorescence quantitative analysis of ERO1L protein indicated that among the 105 patients, 83 were ERO1L positive and 22 were ERO1L negative. The expression of ERO1L in normal adjacent tissues was negative. Further analysis of the association between ERO1L and patient prognosis demonstrated that the cumulative recurrence rate in ERO1L positive patients was significantly higher than that of patients with negative ERO1L. Furthermore, the cumulative survival rate was significantly lower in patients with positive ERO1L than those with negative ERO1L. The aforementioned results indicate that high ERO1L expression is closely associated 
with patient prognosis. Further studies are required to further elucidate the role of ERO1L in gastric cancer.

In conclusion, ERO1L is highly expressed in gastric cancer tissue and is associated with poor prognosis in patients with gastric cancer. ERO1L is expected to become a target for the prevention of gastric cancer.

\section{References}

1. Ye XS, Yu C, Aggarwal A and Reinhard C: Genomic alterations and molecular subtypes of gastric cancers in Asians. Chin J Cancer 35: 42, 2016.

2. Kikuchi S, Kagawa S, Ohara T, Kubota T, Kuwada K, Kagawa T, Kuroda S, Shirakawa Y, Nishizaki M and Fujiwara T: Recurrence after endoscopic curative resection of mucosal gastric cancer associated with an adjacent neoplastic precursor lesion. Acta Med Okayama 70: 213-216, 2016.

3. Davidson M, Okines AF and Starling N: Current and future therapies for advanced gastric cancer. Clin Colorectal Cancer 14 239-250, 2015.

4. Oikawa K, Saito A, Kiyuna T, Graf HP, Cosatto E and Kuroda M: Pathological diagnosis of gastric cancers with a novel computerized analysis system. J Pathol Inform 8: 5, 2017.

5. Zhang B, Han H, Fu S, Yang P, Gu Z, Zhou Q and Cao Z: Dehydroeffusol inhibits gastric cancer cell growth and tumorigenicity by selectively inducing tumor-suppressive endoplasmic reticulum stress and a moderate apoptosis. Biochem Pharmacol 104: 8-18, 2016.

6. Lan F, Zhu M, Qi Q, Zhang Y and Liu Y: Prognostic value of serum tumor abnormal protein in gastric cancer patients. Mol Clin Oncol 5: 216-220, 2016.

7. Zuo Q, Liu J, Zhang J, Wu M, Guo L and Liao W: Development of trastuzumab-resistant human gastric carcinoma cell lines and mechanisms of drug resistance. Sci Rep 5: 11634, 2015.

8. Chen X, Zhao J, Li A, Gao P, Sun J, Song Y, Liu J, Chen P and Wang Z: Clinicopathological significance of claudin 4 expression in gastric carcinoma: A systematic review and meta-analysis. Onco Targets Ther 9: 3205-3212, 2016.

9. Long Q, Zhu X, Wu Y, Feng B, Jin D, Huang J, Lei T, Gan L and Yang Z: Molecular cloning and characterization of the porcine ErolL and ERp44 genes: Potential roles in controlling energy metabolism. Gen Comp Endocrinol 173: 259-269, 2011.

10. Seol SY, Kim C, Lim JY, Yoon SO, Hong SW, Kim JW, Choi SH and Cho JY: Overexpression of endoplasmic reticulum oxidoreductin 1- $\alpha(E R O 1 L)$ is associated with poor prognosis of gastric cancer. Cancer Res Treat 48: 1196-1209, 2016.

11. Periyasamy P, Guo ML and Buch S: Cocaine induces astrocytosis through ER stress-mediated activation of autophagy. Autophagy 12: 1310-1329, 2016.

12. Zhang ZZ, Yuan K, Yue HT, Yuan FH, Bi HT, Weng SP, He JG and Chen YH: Identification and functional characterization of an endoplasmic reticulum oxidoreductin 1- $\alpha$ gene in Litopenaeus vannamei. Dev Comp Immunol 57: 10-19, 2016.

13. Bando E, Makuuchi R, Tokunaga M, Tanizawa Y, Kawamura T and Terashima M: Impact of clinical tumor-node-metastasis staging on survival in gastric carcinoma patients receiving surgery. Gastric Cancer 20: 448-456, 2017.

14. Livak KJ and Schmittgen TD: Analysis of relative gene expression data using real-time quantitative PCR and the 2(-Delta Delta C(T)) Method. Methods 25: 402-408, 2001.
15. Yeldan E, Oguz S, Usta U, Ilhan E and Senlikci A: Risk factors for peritoneal dissemination of gastric cancer. Minerva Chir 70: 91-96, 2015.

16. Zhang WH, Chen XZ, Yang K, Liu K, Guo DJ, Wang W, Zhang B, Chen ZX, Chen JP, Zhou ZG and Hu JK: Risk factors and survival outcomes for postoperative pulmonary complications in gastric cancer patients. Hepatogastroenterology 62: 766-772, 2015.

17. Ji JZ: Review of research on prevention and treatment of gastric cancer in China in thirty years. Chin J Clin Oncol 40: 1345-1351, 2013.

18. Mégraud F, Bessède $\mathrm{E}$ and Varon $\mathrm{C}$ : Helicobacter pylori infection and gastric carcinoma. Clin Microbiol Infect 21: 984-990, 2015.

19. Huang Y, Wu P, Liu B and Du J: Successful personalized chemotherapy for metastatic gastric cancer based on quantitative BRCA1 mRNA expression level: A case report. Oncol Lett 11: 4183-4186, 2016.

20. Li QJ, Shan BE, Li H, Su JW, Zhang C and Li QX: Imbalance of Th17/Treg in patients with gastric cancer. Chin Gen Pract 18: 3596-3601, 2015.

21. Bu X, Zhao Y, Zhang Z, Wang M, Li M and Yan Y: Recombinant Newcastle disease virus (rL-RVG) triggers autophagy and apoptosis in gastric carcinoma cells by inducing ER stress. Am J Cancer Res 6: 924-936, 2016.

22. Seo M, Ryou HJ, Yun EY and Goo TW: Molecular characterization of endoplasmic reticulum Oxidoreductin 1 from Bombyx mori. Int J Mol Sci 16: 26520-26529, 2015.

23. Park S: Defective Anksla disrupts the export of receptor tyrosine kinases from the endoplasmic reticulum. BMB Rep 49: 651-652, 2016.

24. Yang X, Xu H, Hao Y, Zhao L, Cai X, Tian J, Zhang M, Han X, Ma S, Cao J and Jiang Y: Endoplasmic reticulum oxidoreductin $1 \alpha$ mediates hepatic endoplasmic reticulum stress in homocysteine-induced atherosclerosis. Acta Biochim Biophys Sin (Shanghai) 46: 902-910, 2014.

25. Lin S, Zhang J, Chen H, Chen K, Lai F, Luo J, Wang Z, Bu H, Zhang $\mathrm{R}, \mathrm{Li} \mathrm{H}$ and Tong $\mathrm{H}$ : Involvement of endoplasmic reticulum stress in capsaicin-induced apoptosis of human pancreatic cancer cells. Evid Based Complement Alternat Med 2013: 629750, 2013.

26. Huang ZL, Chen RP, Zhou XT, Zhan HL, Hu MM, Liu B, Wu GD and Wu LF: Long non-coding RNA MEG3 induces cell apoptosis in esophageal cancer through endoplasmic reticulum stress. Oncol Rep 37: 3093-3099, 2017.

27. Conti S, Petrungaro S, Marini ES, Masciarelli S, Tomaipitinca L, Filippini A, Giampietri C and Ziparo E: A novel role of c-FLIP protein in regulation of ER stress response. Cell Signal 28: 1262-1269, 2016.

28. Hosoi T and Ozawa K: The mechanisms and pharmacological strategy for treatment of ER stress-induced metabolic syndrome. Yakugaku Zasshi 136: 827-830, 2016.

29. Kawada K, Iekumo T, Kaneko M, Nomura Y and Okuma Y: ER stress-induced aberrant neuronal maturation and neurodevelopmental disorders. Yakugaku Zasshi 136: 811-815, 2016.

30. Enyedi B, Várnai P and Geiszt M: Redox state of the endoplasmic reticulum is controlled by Ero1L-alpha and intraluminal calcium. Antioxid Redox Signal 13: 721-729, 2010.

31. Zou P, Chen M, Ji J, Chen W, Chen X, Ying S, Zhang J, Zhang Z, Liu Z, Yang S and Liang G: Auranofin induces apoptosis by ROS-mediated ER stress and mitochondrial dysfunction and displayed synergistic lethality with piperlongumine in gastric cancer. Oncotarget 6: 36505-36521, 2015. 